\title{
Targeting hepatocellular carcinoma: what did we discover so far?
}

\author{
Ana Filipa Brito, ${ }^{1-3}$ Ana Margarida Abrantes, ${ }^{1-3}$ \\ José Guilherme Tralhão, ${ }^{1-4}$ Maria Filomena Botelho'-3 \\ ${ }^{1}$ Institute of Biophysics and Biomathematics, Institute for Biomedical Imaging and Life Sciences \\ (IBILI), University of Coimbra; ${ }^{2}$ Center of Investigation in Environment, Genetics and Oncobiology, \\ University of Coimbra; ${ }^{3}$ Center for Neuroscience and Cell Biology, Institute for Biomedical Imaging \\ and Life Sciences (CNC. IBILI), University of Coimbra; ${ }^{4}$ Surgical Department, Surgery A, Coimbra \\ Hospital and University Center, Coimbra, Portugal
}

\begin{abstract}
Hepatocellular carcinoma (HCC) is increasingly considered an issue of global importance. Its rates of incidence and mortality have been markedly increasing over the last decades. Among risk factors, some should be highlighted, namely the infections by hepatitis B and $\mathrm{C}$ virus, as well as clinical cases of cirrhosis. HCC is characterized as asymptomatic disease in the initial stages which most often leads to a late diagnosis. At molecular and genetic level HCC represents a highly complex tumor entity, including a wide variety of mutations, thus accounting for different mechanisms of resistance towards therapeutic approaches. In particular, mutations of the TP53 gene, as well as a
\end{abstract}

Correspondence: Ana Filipa Brito, Faculty of Medicine of University of Coimbra, Pólo III - Pólo das Ciências da Saúde, Azinhaga de Santa Comba, Celas. 3000-548 Coimbra, Portugal.

Tel.: +351.239480200 - Fax: +351.239480217 .

E-mail: anabrito816@gmail.com

Key words: Hepatocellular carcinoma; molecular biology; P53.

Acknowledgments: AFB would like to thank the Portuguese Foundation for Science and Technology (FCT) for awarding a PhD scholarship (SFRH/BD/61378/2009). The authors would like to thank the Center of Investigation in Environment, Genetics and Oncobiology (CIMAG0) for financing the project 09/12. The authors thank also FCT, Portugal (Strategic Project PEst-C/SAU/UI3282/2013 and UID/NEU/04539/2013).

Contributions: AFB designed the study, interpreted data and wrote the paper; AMA, JGT and MFB contributed in data interpretation and manuscript writing. All authors read and approved the final manuscript.

Conflict of interest: the authors declare no potential conflict of interest.

Received for publication: 14 July 2016.

Revision received: 19 August 2016.

Accepted for publication: 24 August 2016.

This work is licensed under a Creative Commons Attribution NonCommercial 4.0 License (CC BY-NC 4.0).

CC Copyright A.F. Brito et al., 2016

Licensee PAGEPress, Italy

Oncology Reviews 2016; 10:302

doi:10.4081/oncol.2016.302 deregulation between the expression of pro- and anti-apoptotic proteins of the BCL-2 family are observed. Regarding treatment modalities, surgical procedures offer the best chance of cure, however, due to a late diagnosis, most of concerned patients cannot be subjected to them. Chemotherapy and radiotherapy are also ineffective, and currently, the treatment with sorafenib is the most commonly used systemic therapy although it can only increase the patient survival for some months. In this sense, a quick and accurate investigation is of utmost importance in order to develop ways of early diagnosis as well as new therapies for HCC.

\section{Hepatocellular carcinoma: epidemiology}

Hepatocellular carcinoma (HCC) represents the result of a complex and heterogeneous malignant process that arises in the context of an underlying progressive liver dysfunction. Given its asymptomatic nature in the early stages of the disease, the majority of HCCs is detected in advanced stages of the disease, often leading to incurable clinical situations. ${ }^{1,2}$

HCC often originates from genetic mutations, which alter metabolic pathways and therefore lead the induction of a disordered cell proliferation. These mutations may occur due to various external factors such as virus infections, especially hepatitis $\mathrm{B}$ and $\mathrm{C}$ virus (HBV and HCV) or hepatocellular proliferation associated with chronic hepatitis..$^{2-4}$

Currently HCC accounts for one of the deadliest types of cancer worldwide, representing the second leading cause of cancer deaths. According to GLOBOCAN, in terms of incidence, HCC is the sixth most common cancer, whereby an estimated 782,451 new cases have arisen in 2012. With respect to mortality, HCC is representing the second most lethal type of cancer for men and sixth for women, and, according to GLOBOCAN, in 2012 it is estimated that 745,517 deaths were directly resulting from HCC. . $^{-10}$

HCC incidence varies according to age, ethnicity, gender and geographical distribution. Most cases of HCC (over 80\%) occur in East Asia and sub-Saharan Africa, where incidence rates are greater than 20 per 100,000 individuals. Regarding developed countries, Southern European nations present mean incidence rates between 10 and 20 cases per 100,000, while in Northern Europe, Oceania and the Americas the incidence rates of HCC are low, namely less than 5 cases per $100,000.7,11,12$ HCC rarely appears before 40 years of age, except in populations where HBV infection is hyper endemic. In Europe and North America, the peak of incidence is between 63 and 65 years of age, while in China, one of the world's major focuses of HCC, the median age at diagnosis is between the 55 and 59 years. ${ }^{11-13}$ 
The HCC risk of incidence is 2-7 times higher in men than in women, varying according to the region of the globe. There are three main reasons that lead to this difference in incidence according to gender. First, males are in general more commonly exposed to hepatitis virus and liver carcinogenic elements, such as tobacco and alcohol consumption. Second, women appear to have a lower predisposition towards developing HCC, once estrogen might have the effect of suppressing inflammation mediated by interleukin 6 , which reduces hepatic damage and compensatory proliferation. Furthermore, in man, it is known that testosterone can induce an increase in androgen receptor signaling, which in turn promotes HCC cells proliferation. $7,11,14$

The main risk factors for developing this type of cancer are $\mathrm{HBV}$ and HCV infection followed by alcoholism and smoking. In Africa and Asia in particular, the ingestion of foods containing mycotoxins, specifically aflatoxin B (AFB1) has to be taken into account. Moreover, hepatic cirrhosis, related to both, chronic alcohol ingestion and HBV, as well as $\mathrm{HCV}$, is equally considered representing one of the main risk factors for HCC development. It is considered that approximately $80 \%$ of patients with HCC develop cirrhosis before the emergence of the tumor. ${ }^{15}$

The differences in incidence rates of HCC in different regions of the world are a reflection of the geographical distribution of risk factors for this type of cancer. It is noted that countries with high prevalence of infection by HBV and HCV are those that have higher incidence rates of HCC. In agreement with these data is the fact that approximately $75 \%$ of HCC cases are due to chronic infections by these viruses. ${ }^{4,7}$

Among the risk factors HBV infection is highlighted as the most common cause of HCC. More than half of the population diagnosed with HCC is infected with HBV. ${ }^{16}$ HBV belongs to the family of hepadnaviruses and is capable of integrating the human genome, which can activate proto-oncogenes or suppressing cell regulatory genes, allowing the hepatocytes to enter into processes which lead to malignant transformation. ${ }^{417}$ However, it is known that the risk of developing HCC in patients suffering from hepatitis B differs according to ethnicity. African and Asian individuals develop HCC during adult age and not always associated with cirrhosis, while Caucasians develop liver tumors at a later age, usually after a period of progressive liver cirrhosis. This disparity can be explained by both genetic variations between ethnic groups, and through the age difference that populations have at the time of HBV infection. ${ }^{7}$ From the HCCs originated by HBV, $70 \%$ to $90 \%$ arise in cirrhotic patients. However, this virus is also considered the leading cause of cirrhosis in the absence of HCC.11

HCV infection is also a major risk factor for HCC development. Nevertheless, due to the scarcity of long-term data, the probability of virus-infected people to develop HCC is currently still difficult to determine, representing an estimated $1 \%$ to $3 \%$ after 30 years. HCV also promotes the development of fibrosis and eventually cirrhosis which increases the risk of HCC development. ${ }^{18}$ Some studies also indicate that people co-infected with HIV and HBV or HCV may develop liver diseases, such as cirrhosis or HCC in a much faster way than without viral co-infections. , $^{46,19,20}$

Apart from infections with hepatitis viruses, it is also known that food contamination with AFB1 represents a major risk factor for HCC development. Furthermore, it is now known that AFB1 can act in synergy with $\mathrm{HBV}$ in the pathogenesis of this type of tumor. AFB1 induces DNA mutations, particularly in codon 249 of TP53 with mitigation of its tumor suppressor function. In Asian and African regions, efforts have been developed to eliminate the food exposure to this toxin. .,17 $^{7}$

There are others risk factors which, although having a smaller role, contribute to HCC development. Among these are diabetes mellitus, nonalcoholic steatohepatitis and dietary factors, such as chronic alcohol ingestion, which often lead to cirrhosis, representing an important risk factor by itself. ${ }^{19,21,22}$ Obesity also appears to contribute to the development of this neoplasia either isolated or in synergy with the related diabetes mellitus constituting a risk factor. However, the molecular mechanism by which obesity and/or diabetes mellitus induce the HCC development is not yet fully characterized. Nevertheless, it is known that the hepatic inflammation arising from cases of diabetes and/or cirrhosis induces oxidative stress and lipid peroxidation, which in turn leads to hepatic fibrosis and eventually cirrhosis, which may constitute a mechanism of HCC development in patients suffering from those pathologies. More recently, some studies have shown the existence of a synergistic interaction between alcohol, diabetes, and viral hepatitis which induce severe consequences in HCC development. ${ }^{7,23}$ Finally, it is also known that there are some genetic and metabolic diseases such as hemochromatosis, Wilson's disease, porphyria and 1antitrypsin deficiency which can be risk factors for the HCC development. ${ }^{24}$

\section{Molecular and genetic alterations}

At the genetic level HCC cells are described as having significant changes in a variety of chromosomes. Until now several mutations in different genes that are reflected in the proteins encoded by them, have been found. Among the proteins involved there are to highlight the $\mathrm{P} 53,{ }^{9,15,25} \mathrm{P} 73,{ }^{15,26} \mathrm{P} 16,{ }^{15,26}$ retinoblastoma protein, ${ }^{15,27} \mathrm{APC},{ }^{15}$ PTEN, ${ }^{15,28}$ BRCA2, ${ }^{15,29}$ SMAD2 and $-4,{ }^{15}$ DLC- $1,{ }^{15}$ IGF-2, ${ }^{15,30}$ SOCS- $1,{ }^{15} \beta$ catenin, ${ }^{15,26}$ c-myc ${ }^{15,26,27}$ and cyclin D1. ${ }^{15}$

Of the genes commonly mutated and consequent proteins abnormally expressed in HCC cells one of particular importance is the TP53 gene (Figure 1) and its corresponding protein, the P53. ${ }^{31,32}$

In addition to the mutations in specific genes, there are also several signaling pathways and molecular mechanisms involved in HCC development. These include the mechanisms that regulate cell proliferation and survival, angiogenic factors produced by tumor cells, receptors of oncogenes, as well as growth factors and their receptors. Among these different mechanisms the over-expression of epidermal growth factor (EGF) (Figure 1) with a possible stimulation of receptor tyrosine kinase is noteworthy. In turn, this stimulation keeps the receptor active, thereby stimulating the transduction of the cell membrane information to the nucleus. There are other mechanisms and second messengers that are involved in the HCC development, conducting molecules and transmitting signals from the cell surface to the nucleus. One of these pathways is the MAPK pathway (Figure 1), which acts

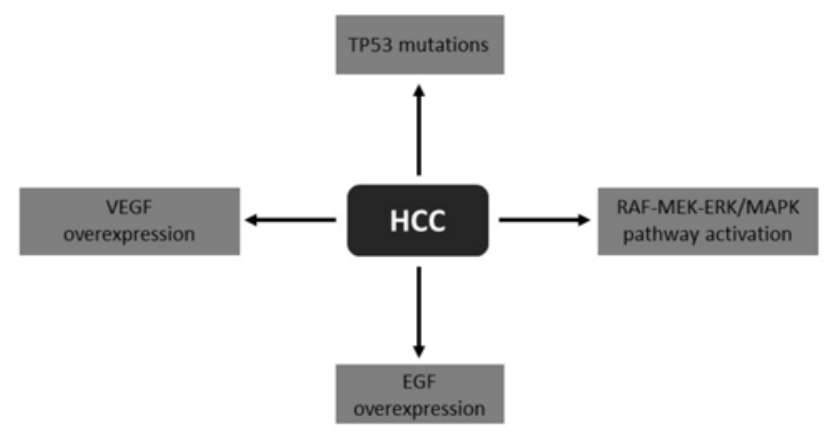

Figure 1. Schematic representation of the main molecular and genetic alterations present in hepatocellular carcinoms (HCC). In many cases of HCC are present mutations in the TP53 gene which induces changes in the expression of the corresponding protein P53. It is also quite common to observe an over expression of vascular endothelial growth factor (VEGF) and epidermal growth factor (EGF), as well as a RAF-MEK-ERK/MAPK signaling pathway activation. 
through the RAS protein. This protein is activated by the binding action of a growth factor to membrane receptors subsequently undergoing autophosphorylation, which allows it to transmit the signal through activation of other components of this signaling pathway such as ERK1, RAF and MEK (Figure 1). ${ }^{15,33,34}$

Angiogenesis also has a very active role in HCC development, as it is involved in the stimulation of cell proliferation. HCC is a highly vascularized tumor, and the vascular endothelial growth factor (VEGF) is widely expressed and particularly active in this type of cancer (Figure 1). In addition, there is a close connection between tumor angiogenesis and the referred MAPK pathway, since the number of receptors required for this signaling cascade increases in response to angiogenesis, contributing to differentiation, regulation of cell proliferation and tumor survival. ${ }^{15,33,34}$

\section{Deregulation between survival and cell death in hepatocellular carcinoma}

Apoptosis is a fundamental process in carcinogenesis and in response to anti-cancer treatment. In this regard, it can be noted that deficiencies in the apoptosis activation have been correlated with the development and progression of liver tumors. ${ }^{35}$ P53 has a key role in this process. It is known that among the most common molecular changes observed in HCC are mutations in the TP53 gene and in the P53 protein. ${ }^{31,32,35,36}$ Under normal conditions, P53 is present in the cells in low concentrations but transcriptional activation of this protein may occur in response to various stimuli including DNA damages, hypoxia or deregulated cell cycle progression. P53 protein has several important biological functions including the maintenance of the cell cycle, repair of DNA damage, the balance between cell proliferation and apoptosis, the response to cellular stress and inhibition of angiogenesis. 35,36

Furthermore, the cell cycle regulation is one of the P53 main functions. For stabilizing the genome, P53 can act either directly or indirectly. In a direct form, this protein is able to induce cell cycle arrest in the Gl phase which can be reversible and beneficial to cells re-integration, due to that fact that, in response to damage, such as chemical mutagenic action, P53 can induce apoptosis or promote DNA repair contributing to the genomic stability. Indirectly, P53 can act as a transcriptional activator of several genes inducing the expression and/or activation of the corresponding proteins, which in turn have different cellular functions. Among them TP21 should be noted (Figure 2). The P21 protein is able to inhibit the growth of a wide variety of cells including tumor cells, since P21 inhibits the cyclin-dependent kinases action which are required for the conduct of the cell cycle. In addition to TP21, several other genes are P53 targets in this context, such as the cyclin G and 14th-3-3б.37,38 Meanwhile, there are also genes capable of inhibiting P53, in particular MDM2, the main regulator of P53 which is amplified in approximately $7 \%$ of all human cancers. The MDM2 and P53 are regulated by a feedback mechanism (Figure 2). On one hand, the MDM2 gene promotes the phosphorylated degradation of P53, thus inhibiting its function as a transcriptional activator, on the other hand, the P53 has the ability to activate MDM2 protein. ${ }^{37,39}$

It should also be noted that P53 has a major role in the activation of signaling pathways leading to apoptosis. However, in addition to P53, a large number of target genes of this protein are involved in these signaling pathways, where the pro-apoptotic protein BAX is found, which is able to form heterodimers together with BCL-2 in order to maintain homeostasis. P53 has the ability to induce the expression of BAX (Figure 2) and therefore activating the intrinsic apoptosis pathway. ${ }^{36}$ Furthermore, it is known that the cells that present P53 mutated, a recurring fact on HCC, generally have a longer life and are more resist- ant to chemotherapy and radiotherapy. ${ }^{25,40}$ However, although it is known that mutations in the TP53 gene are common in HCC, and that altered expression of P53 can be linked to tumor progression, advanced tumor grade, survival and response to therapy, little is known about how mutations in TP53 gene correlate with HCC recurrence. ${ }^{38}$ With regard to therapy, it is known that most drugs used in chemotherapy require the action of P53 to induce apoptosis, and thus, tumors with low or mutated expression of this protein are generally resistant to chemotherapy, which is one of the main mechanisms which explain the low efficiency of conventional chemotherapy in HCC. ${ }^{41}$

The majority of genetic modifications observed in HCC induce an imbalance between the expression of pro and anti-apoptotic proteins of BCL-2 family, which could be correlated with changes in the TP53 gene expression. It is known, that the expression of anti-apoptotic members of this family, such as BCL-XL and MCL-1 is increased in a large percentage of HCCs. In contrast, the expression of some pro-apoptotic members of this family, such as BAX and BCL-XS is decreased in HCCs that express a modified P53. ${ }^{35,41-44}$

Some studies also revealed that in about $90 \%$ of advanced cases, HCCs exhibit high expression levels of XIAP, a well-known inhibitor of caspases. ${ }^{35}$ In this way, XIAP over-expression can offer resistance to the evasion of apoptosis in HCC. Additionally, survivin, representing another member of inhibitors of the apoptosis family, is over-expressed in certain HCC cell lines and tissues. Apparently, survivin appears to have an important role in the cancer progression by inhibiting apoptosis and also promoting cell proliferation. In this context, some studies indicate that this molecule can be correlated with a poor prognosis and a high risk of recurrence. $35,41,45$

There are also many other factors that lead to the high resistance of HCC to apoptosis. The disruption in the TGF- $\beta$ signaling pathway, which induces deregulation of apoptosis, is equally noteworthy. It is now confirmed that the TGF- $\beta$ expression is increased in a large proportion of patients with HCC, however, some results indicate that this over-expression may play a dual role in the control of apoptosis either in hepatocytes or in HCC, in other words, on one hand, TGF- $\beta$ induces cell death, but otherwise can activate anti-apoptotic stimuli. ${ }^{41,46}$ Apparently, in HCC cells the effect of anti-apoptotic stimuli activation through this mechanism seems to be active, contributing to tumor pro-

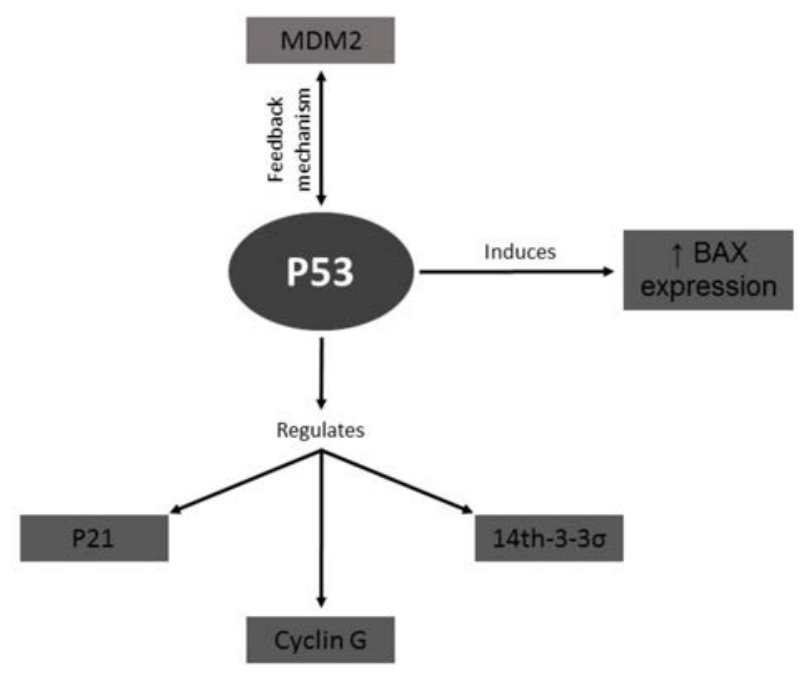

Figure 2. Schematic representation of some of the major effects that the P53 has on proteins involved in cell death pathways. It is noted the occurrence of a feedback mechanism between P53 and MDM2. It is also observed that the P53 regulates the expression of P21, cyclin G and 14th-3-3 $\sigma$ well as induces a BAX overexpression. 
gression and consequent invasion. ${ }^{35,41,46}$ Apoptosis resistance presented HCC is also mediated by different receptors of death. Through the expression of FAS and FAS ligand it is possible to predict HCC recurrence. It is also known that the cFLIP, an intracellular inhibitor of caspase 8 activation, is constitutively expressed in human HCC cell lines and shows increased levels in tumor and non-tumor liver tissues, offering, in this way resistance to the extrinsic pathway of apoptosis. ${ }^{35,41}$ The over-expression of BRE has also been described in tissues of HCCs. BRE is an anti-apoptotic protein which binds to the cytoplasmic domains of tumor necrosis factor and FAS, in such fashion attenuating apoptosis. ${ }^{35,41,45,47}$ TRAIL selectively induces apoptosis in a variety of transformed cell lines, but almost never in normal tissues. In most cases, HCC cells express TRAIL protein and mRNA, and some data indicate that most of the HCC cells are insensitive to TRAIL-mediated apoptosis..$^{35,47}$

Overexpression of survival signaling pathways by HCC cells is itself an obstacle to apoptosis. Some activators of autocrine signals such as EGF receptor (EGFR) ligands, can protect primary liver tumors cells from apoptosis induced by stress, by physiological factors or pro-apoptotic drugs. Further, deregulation of growth factors signaling pathways including EGF pathways and IGF-1 has been well established in human HCCs. The P60C-src is also overexpressed in HCC cells, protecting these cells from death stimuli and inhibiting their sensitivity to TRAIL. ${ }^{35,48}$ The PI3K/AKT signaling pathway is also modified in HCC cells, and PTEN gene expression is absent or else present in a very residually form in more than half of these types of tumors. ${ }^{35,41,49}$

Thus, there are numerous molecular alterations that contribute to apoptosis resistance observed in HCCs. However, it is noted that most of the observed genetic alterations lead to deregulation in the balance on the expression of pro and anti-apoptotic proteins of the BCL-2 family. More specifically it is known that the expression of pro-apoptotic members of this family such as BAX or BCL-XS is decreased in liver tumors with P53 mutations. This finding greatly strengthens the role that BCL-2 family proteins as well as the tumor suppressor gene TP53 have in HCC development and response to therapy, indicating that new therapeutic strategies based on these genes and/or proteins, can be extremely useful in the treatment of this type of tumor.

\section{Treatment}

Among the therapeutic options, liver transplantation is the most effective therapy because it allows simultaneous treatment of cancer and associated liver disease. However, only about 5\% of patients can benefit from this therapy, since among other obstacles are the compatibility and the availability of organs. Furthermore, transplantation can only be applied to patients with HCC in the initial stage of decompensated cirrhosis. According to the Milan criteria, transplantation is usually performed only in patients with isolated tumors smaller than $5 \mathrm{~cm}$, or up to three nodules smaller than $3 \mathrm{~cm} .{ }^{50,51}$ However, after transplantation in addition to possible organ rejection other complications such as viral reinfection may occur, and in $50 \%$ of cases 5 years after transplantation patients develop cirrhosis. ${ }^{52-55}$ Surgical resection by partial hepatectomy is also a curative therapeutic option. However, this surgical procedure is only feasible in patients without associated major liver disease (Child-Pugh score B and C or model for end-stage liver disease $>10$ ). The survival rate of patients undergoing this therapy is between $60 \%$ and $70 \%$ after 5 years, however the high rate of the disease recurrence is the main disadvantage of the partial liver resection. The recurrence rate may be due to the formation of a new HCC, or the presence of microvascular invasion or satellite nodules..$^{51-55}$

Although surgical treatment modalities provide the best results, the majority of patients at diagnosis may not benefit from these therapeu- tic options. In this case, the treatment options most frequently used are radiofrequency, alcoholization or chemoembolization. These techniques are performed with imaging control, and its maximum efficiency is for nodules less than $3 \mathrm{~cm}$ of diameter, for which the complete response rate is around $80 \%$. However, these techniques also imply disadvantages, whereby a high number of recurrences has to be emphasized. ${ }^{52-56}$ Specifically transarterial chemoembolization involves injection of a cytotoxic drug combined with lipiodol, for example, or with microspheres followed by embolization with the use of absorbable particles, allowing high doses of local chemotherapy, which in turn leads to reduction rate of tumor progression, with objective responses between $16 \%$ and $55 \%$ of patients. ${ }^{16,53,56-58}$

Finally, although with disappointing results there are systemic chemotherapy and radiotherapy. However, due to poor results, its use is controversial and only applicable in the context of palliative treatment. With respect to chemotherapy, HCC is usually classified as a highly chemoresistant tumor, with minimal efficacy of systemic chemotherapy. Nevertheless, taking into account that the vast majority of patients cannot take advantage of the potentially curative therapies described above, conventional chemotherapy is still used in a palliative treatment form for the purpose of attenuating the symptoms resulting from the disease and improving the patients quality of life. Among the drugs used in systemic chemotherapy for HCC treatment, doxorubicin is one of the most used, although only a partial response is observed in less than $10 \%$ of patients and it does not increase overall survival. On the other hand, the administration of 5 -fluorouracil in combination therapy is controversial, although it is defended by some authors. Cisplatin equally continues to be used in the treatment of this neoplasia, either alone or in combination therapy. $9,42,59$

Besides the mentioned drugs, the therapeutic efficacy of other drugs often used in chemotherapy such as mitoxantrone, placlitaxel, irinotecan, gemcitabine and capecitabine, both alone and in combination have been studied in HCC treatment. However, the results obtained are equally disappointing. ${ }^{43,44,60}$

Regarding radiotherapy, it plays a minor role in HCC treatment, although it can be also used. The liver and its primary tumors are highly radioresistant, which leads to the ineffectiveness of this type of treatment. Additionally, the molecular heterogeneity of hepatic tumors, as well as the expression of the P53 gene and other genes and/or proteins involved in cell death and proliferation pathways might provide the

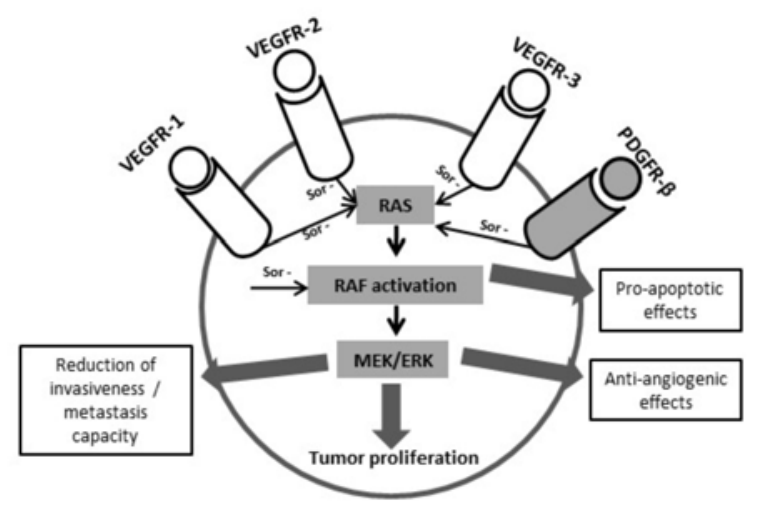

Figure 3. Several molecular targets of sorafenib. Sorafenib inhibits the autophosphorylation of vascular endothelial growth factor receptor (VEGFR)-1, VEGFR-2, VEGFR-3 and plateletderived growth factor receptor (PDGFR)- $\beta$, as well as prevents RAF activation. Inhibition of RAF is associated with downregulation of some downstream signaling pathways and other molecular pathways leading to apoptosis. 
basis for the development of resistance and/or sensitivity towards radiation. 61,62

So today, the beam radiation therapy for HCC is usually limited to palliative treatment of metastasis of HCC. ${ }^{63}$ Nevertheless, given the technological advances the use of radiotherapy (in its various forms) for treatment of advanced HCC has been growing, with promising results. Internal radiation therapy with Yttrium-90 ${ }^{90} \mathrm{Y}$ ) (radioembolization) has been increasingly used in the treatment of unresectable HCC by injecting microspheres via the hepatic artery. The arterial administration of ${ }^{90} \mathrm{Y}$ beads allows localized application of high doses of radiation in HCC cells with sparing of adjacent normal tissues. ${ }^{63}$ Similarly other radioisotopes have also been used in the treatment of HCC. Are examples Holmium-199 $\left({ }^{199} \mathrm{Ho}\right)$ in chitosan complex and Iodine-131 ( $\left.{ }^{131} \mathrm{I}\right)$ as ${ }^{131}$ I-lipiodol. ${ }^{32,63}$ More recently, the development of more advanced technologies such as stereotactic body radiotherapy (SBRT) has introduced new hope for the treatment of HCC using radiation. The SBRT is a technique that uses multiple beams forming to deliver high doses of radiation with a rapid fall-off beyond the target volume. The molecular mechanisms underlying cell death induced by SBRT are not yet fully known but it is believed that in addition to DNA damage and cell death been observed in fractional conventional radiotherapy, high doses of radiation resulting from this technique also results in an ablative effect tumor through a vascular injury. ${ }^{63,64}$

The development of specific therapies for some molecular targets came to provide major advances in cancer therapy. 65,66 One of the signaling pathways that is often deregulated in solid tumors, including HCC is, as already referenced, the RAF-MEK-ERK/MAPK pathway. Several studies have sought to develop a molecule that interferes with this target, which led to the discovery of sorafenib in 1990. Later it was found that sorafenib acts not only on the tumor itself, but also in the microvasculature and is therefore considered a double-acting multikinase inhibitor.

Namely, it is now known that this drug inhibits cell proliferation through a strong inhibition of serine/threonine kinases RAF, more particularly the RAF-1, the RAF-B wild type, the RAF-B oncogenic V600E the MAPK signaling pathway (Figure 3). Moreover, this drug inhibits in a highly effective way the pro-angiogenic VEGF receptors (VEGFR)-1, VEGFR-2, VEGFR-3 and the platelet-derived growth factor receptor$\beta^{65,66}$ In parallel, it has been shown that the sorafenib is capable of inducing apoptosis in a wide variety of tumor cell lines, though the mechanism underlying this induction is not yet fully understood. It is known however that sorafenib inhibits the phosphorylation of eIF4E which results in decreased expression of anti-apoptotic protein MCL-1. In turn, the sub-expression of MCL-1 induces the release of cytochrome c from the mitochondria to the cytoplasm, which in turn activates caspases and induces apoptotic cell death. ${ }^{65,66}$

In effective terms, sorafenib was approved by the U.S. Food and Drug Administration for the treatment of metastatic renal cell carcinoma in December 2005, obtaining authorization for marketing by the European Union for the same pathology in the following year. ${ }^{67}$ Later it was also approved for the treatment of HCC and metastatic differentiated thyroid carcinoma resistant to metabolic radiotherapy with ${ }^{131} \mathrm{I} .{ }^{67}$ Similar to what happens with other drugs used in the treatment of cancer also treatment with sorafenib may induce some adverse effects of which the main ones are: hand-foot skin reaction, rash/desquamation, dyspepsia, diarrhea, fatigue, hypertension, thromboembolic and cardiac ischemic events. ${ }^{67}$

Regarding HCC, this type of tumor is markedly hypervascular and several studies have demonstrated the existence of a VEGFR overexpression in human HCC samples. ${ }^{68}$ However, although it appears that the sorafenib mechanism of action in HCC is at the vascularization level, it should not be taken as a single because there are studies that highlight the role of sorafenib on RAF pathway inhibition in the HCC treatment. ${ }^{69,70}$ It is now known that both RAF/MEK/ERK signaling cascade as angiogenesis play critical roles in the HCC development and, therefore, in this kind of tumor, sorafenib can act both at the level of cancer cell, either at the level of neovascularization (Figure 3). ${ }^{65,66,70}$ However, even if treatment with sorafenib is currently regarded as the best option in HCC, it is only able to prolong patients' survival for a couple of months.

In the era of targeted therapies, the effect of various other drugs in the treatment of HCC has been widely studied. Examples are drugs that act on angiogenesis, such as sunitinib, brivanib, linifanib, vatalanib, TSU-68, cediranib, bevacizumab and ramucirumab. ${ }^{71}$ Furthermore were also conducted with cetuximab (anti-EGFR antibody) with erlotinib and lapatinib (EGFR inhibitors) with selumetinib (MEK $1 / 2$ competitive inhibitor), with everolimus (inhibitor of mTOR) and with tivantinib (c-MET inhibitor). ${ }^{71}$ Also multikinase inhibitors been the subject of intensive study with respect to the treatment of HCC, examples are nintedanib and regorafenib. ${ }^{71}$ Although some studies with these drugs have already arrived clinical trials in phases II and III, the reality is that to date none of them proved to be quite effective in the treatment of HCC. ${ }^{71}$ So the reality is that the prognosis of HCC patients remains bleak. Thus, new therapeutic targets as well as new drugs and/or therapeutic modalities are urgently needed, allowing us to more effectively combat this complicated type of tumor.

\section{Conclusions}

Nowadays, HCC should be considered a problem of global importance. Due to the lack of a timely diagnosis, the majority of HCC patients will most likely not be able to benefit from surgical treatments (total or partial hepatic resection), therefore being conducted to other therapeutic modalities. However, at the genetic level HCC cells are described as having significant variations in several chromosomes, leading to mutations in genes that are normally involved in apoptosis, such as TP53. This genetic instability makes HCC commonly resistant to the surgery alternatives, such as radiation and chemotherapy and in this sense, these treatments in this type of tumor, when used, have only a palliative nature. More recently, a widespread use of sorafenib as double-acting multikinase inhibitor for the treatment of HCC has been introduced. However, even sorafenib treatment allows only the increase of patients' survival in a few months. Thus, the incidence and mortality from HCC have very similar values, leading to an urgently searching need for new forms of diagnosis and treatment of HCC.

\section{References}

1. Finn RS. Emerging targeted strategies in advanced hepatocellular carcinoma. Semin Liver Dis 2013;33:S11-9.

2. Gomes MA, Priolli DG, Tralhão JG, Botelho MF. Hepatocellular carcinoma: epidemiology, biology, diagnosis, and therapies. Rev Assoc Med Bras 2013;59:514-24.

3. Singal A, El-Serag H. Hepatocellular carcinoma from epidemiology to prevention: translating knowledge into practice. Clin Gastroenterol Hepatol 2015;13:2140-51.

4. Sukowati CHC, El-khobar KE, Ie SI, et al. Significance of hepatitis virus infection in the oncogenic initiation of hepatocellular carcinoma. World J Gastroenterol 2016;22:1497-512.

5. Jemal A, Bray F, Center MM, et al. Global cancer statistics. CA Cancer J Clin 2011;61:69-90.

6. Venook AP, Papandreou C, Furuse J, de Guevara LL. The incidence and epidemiology of hepatocellular carcinoma: a global and region- 
al perspective. Oncologist 2010;15:5-13.

7. Yang JD, Roberts LR. Hepatocellular carcinoma: a global view. Nat Rev Gastroenterol Hepatol 2010;7:448-58.

8. Ferlay J, Shin HR, Bray F, et al. Estimates of worldwide burden of cancer in 2008: GLOBOCAN 2008. Int J Cancer 2010;127:2893-917.

9. Brito AF, Abrantes AM, Pinto-Costa C, et al. Hepatocellular carcinoma and chemotherapy: the role of p53. Chemotherapy 2012;58:381-6.

10. International Agency for Research on Cancer (IARC). GLOBOCAN 2012: Estimated cancer incidence, mortality and prevalende worldwide in 2012. Available from: http://globocan.iarc.fr/Default.aspx

11. El-Serag HB. Epidemiology of viral hepatitis and hepatocellular carcinoma. Gastroenterology 2012;142:1264-73.

12. Zhu RX, Seto W, Lai C, Yuen M. Epidemiology of hepatocellular carcinoma in the Asia-Pacific region. Gut Liver 2016;10:332-9.

13. Schütte K, Bornschein J, Malfertheiner P. Hepatocellular carcinoma-epidemiological trends and risk factors. Dig Dis 2009;27:8092.

14. Shi L, Feng Y, Lin H, et al. Role of estrogen in hepatocellular carcinoma: is inflammation the key? J Transl Med 2014;12:1-9.

15. Alves RCP, Alves D, Guz B, et al. Advanced hepatocellular carcinoma. Review of targeted molecular drugs. Ann Hepatol 2011;10:21-7.

16. Bruix J, Sherman M. Management of hepatocellular carcinoma. Hepatology 2005;42:1208-36.

17. Mazzanti R, Gramantieri L, Bolondi L. Hepatocellular carcinoma: epidemiology and clinical aspects. Mol Aspects Med 2008;29:13043.

18. El-Serag HB, Rudolph KL. Hepatocellular carcinoma: epidemiology and molecular carcinogenesis. Gastroenterology 2007;132:2557-76.

19. Neuveut C, Wei Y, Buendia MA. Mechanisms of HBV-related hepatocarcinogenesis. J Hepatol 2010;52:594-604.

20. Tralhao JG, Roudier J, Morosan S, et al. Paracrine in vivo inhibitory effects of hepatitis B virus $\mathrm{X}$ protein $(\mathrm{HBx})$ on liver cell proliferation: an alternative mechanism of $\mathrm{HBx}$-related pathogenesis. Proc Natl Acad Sci U S A 2002;99:6991-6.

21. Blonski W. Non-viral causes of hepatocellular carcinoma. World J Gastroenterol 2010;16:3603.

22. Brechot C, Kremsdorf D, Soussan P, et al. Hepatitis B virus (HBV)related hepatocellular carcinoma (HCC): molecular mechanisms and novel paradigms. Pathol Biol (Paris) 2010;58:278-87.

23. Yu MC, Yuan JM. Environmental factors and risk for hepatocellular carcinoma. Gastroenterology 2004;127:S72-S78.

24. Wang XW, Hussain SP, Huo TI, et al. Molecular pathogenesis of human hepatocellular carcinoma. Toxicology 2002;27:43-7.

25. Guan Y, He Q, La Z. Roles of p53 in carcinogenesis, diagnosis and treatment of hepatocellular carcinoma. J Cancer Mol 2006;2:191-7.

26. Qin L, Tang Z. The prognostic molecular markers in hepatocellular carcinoma. World J Gastroenterol 2002;8:385-92.

27. Iakova P, Timchenko L, Timchenko N. Intracellular signaling and hepatocellular carcinoma. Semin Cancer Biol 2012;21:28-34.

28. Shearn C, Petersen D. Understanding the tumor suppressor PTEN in chronic alcoholism and hepatocellular carcinoma. Adv Exp Med Biol 2015;815:173-84.

29. Kondoh N, Wakatsuki T, Hada A, et al. Genetic and epigenetic events in human hepatocarcinogenesis. Int J Oncol 2001;18:1271-8.

30. Enguita-germán M, Fortes P. Targeting the insulin-like growth factor pathway in hepatocellular carcinoma. World $\mathrm{J}$ Hepatol 2014;6:716-37.

31. Brito AF, Abrantes AM, Ribeiro M, et al. Fluorine-18 fluorodeoxyglucose uptake in hepatocellular carcinoma: correlation with glucose transporters and p53 expression. J Clin Exp Hepatol 2015;5:183-9.

32. Gomes AR, Abrantes AM, Brito AF, et al. Influence of P53 on the radiotherapy response of hepatocellular carcinoma. Clin Mol Hepatol 2015;21:257-67.

33. Villanueva A, Llovet JM. Targeted therapies for hepatocellular carci- noma. Gastroenterology 2011;140:1410-26.

34. Delire B, Starkel P. The Ras/MAPK pathway and hepatocarcinoma: pathogenesis and therapeutic implications. Eur J Clin Invest 2015;45:609-23.

35. Fabregat I. Dysregulation of apoptosis in hepatocellular carcinoma cells. World J Gastroenterol 2009;15:513.

36. Ferreira CG, Tolis C, Giaccone G. P53 and chemosensitivity. Ann Oncol 1999;10:1011-21.

37. Tokino T, Nakamura Y. The role of p53-target genes in human cancer. Crit Rev Oncol Hematol 2000;33:1-6.

38. Chen GG, Merchant JL, Lai PBS, et al. Mutation of p53 in recurrent hepatocellular carcinoma and its association with the expression of ZBP-89. Am J Pathol 2003;162:1823-9.

39. Forslund A, Zeng Z, Qin LX, et al. MDM2 gene amplification is correlated to tumor progression but not to the presence of SNP309 or TP53 mutational status in primary colorectal cancers. Mol Cancer Res 2008;6:205-11.

40. Akimoto M, Yoshikawa M, Ebara M, et al. Relationship between therapeutic efficacy of arterial infusion chemotherapy and expression of P-glycoprotein and p53 protein in advanced hepatocellular carcinoma. World J Gastroenterol 2006;12:868-73.

41. Fabregat I, Roncero C, Fernández M. Survival and apoptosis: a dysregulated balance in liver cancer. Liver Int 2007;27:155-62.

42. Johnson PJ. Systemic chemotherapy of liver tumors. Semin Surg Oncol 2000;19:116-24.

43. Giglia JL, Antonia SJ, Berk LB, et al. Systemic therapy for advanced hepatocellular carcinoma: past, present, and future. Cancer Control 2010;17:120-9.

44. Yeo W, Mok TS, Zee B, et al. A randomized phase III study of doxorubicin versus cisplatin/interferon alpha-2b/doxorubicin/fluorouracil (PIAF) combination chemotherapy for unresectable hepatocellular carcinoma. J Natl Cancer Inst 2005;97:1532-8.

45. Eichhorst S. Modulation of apoptosis as a target for liver disease. Exp Opin Ther Targets 2005;9:83-99.

46. Giannelli G, Mikulits W, Dooley S, et al. The rationale for targeting TGF-b in chronic liver diseases. Eur J Clin Invest 2016;46:349-361.

47. Cullen SP, Martin SJ. Seminars in cell and developmental biology FAS and TRAIL "death receptors" as initiators of inflammation: implications for cancer. Semin Cell Dev Biol 2015;39:26-34.

48. Asaki TSM, Kada MA0, Hiratori YAS, et al. pp60 c-src activation in hepatocellular carcinoma of humans and LEC rats. Hepatology 1998;7:1257-64.

49. Shearn C, Petersen D. Understanding the tumor suppressor PTEN in chronic alcoholism and hepatocellular carcinoma. Adv Exp Med Biol 2015;815:173-84.

50. Mazzaferro V, Regalia E, Doci R, et al. Liver transplantion for the treatment of small hepatocellular carcinomas in patients with cirrhosis. N Engl J Med 1996;334:693-9.

51. Pascual S, Herrera I, Irurzun J, et al. New advances in hepatocellular carcinoma. World J Hepatol 2016;8:421-38.

52. Bruix J, Sherman M. Management of hepatocellular carcinoma: an update. Hepatology 2011;53:1020-2.

53. Bruix J, Boix L, Sala M, Llovet JM. Focus on hepatocellular carcinoma. Cancer Cell 2004;5:215-9.

54. Wörns MA, Galle PR. Future perspectives in hepatocellular carcinoma. Dig Liver Dis 2010;42:S302-9.

55. Wörns MA, Weinmann A, Schuchmann M, Galle PR. Systemic therapies in hepatocellular carcinoma. Dig Dis 2009;27:175-88.

56. Llovet JM. Updated treatment approach to hepatocellular carcinoma. J Gastroenterol 2005;40: 225-35.

57. Bruix J, Llovet JM. Prognostic prediction and treatment strategy in hepatocellular carcinoma. Hepatology 2002;35:519-24.

58. Raoul JL. Natural history of hepatocellular carcinoma and current treatment options. Semin Nucl Med 2008;38:S13-8. 
59. Brito AF, Mendes M, Abrantes AM, et al. Positron emission tomography diagnostic imaging in multidrug-resistant hepatocellular carcinoma: focus on 2-deoxy-2-(18F)fluoro-D-glucose. Mol Diagn Ther 2014;5:495-504.

60. Hussain SP, Schwank J, Staib F, et al. TP53 mutations and hepatocellular carcinoma: insights into the etiology and pathogenesis of liver cancer. Oncogene 2007;26:2166-76.

61. Ma S, Jiao B, Liu X, et al. Approach to radiation therapy in hepatocellular carcinoma. Cancer Treat Rev 2010;36:157-63.

62. Cao Y, Fu YL, Yu M, et al. Human augmenter of liver regeneration is important for hepatoma cell viability and resistance to radiationinduced oxidative stress. Free Radic Biol Med 2009;47:1057-66.

63. Kalogeridi M, Zygogianni A, Kyrgias G, et al. Role of radiotherapy in the management of hepatocellular carcinoma: a systematic review. World J Hepatol 2015;7:101-12.

64. Keane F, Wo J, Zhu A, Hong T. Liver-directed radiotherapy for hepatocellular carcinoma. Liver Cancer 2016;5:198-209.

65. Ibrahim N, Yu Y, Walsh WR, Yang JL. Molecular targeted therapies for cancer: sorafenib mono-therapy and its combination with other therapies (review). Oncol Rep 2012;27:1303-11.

66. Wilhelm SM, Adnane L, Newell P, et al. Preclinical overview of sorafenib, a multikinase inhibitor that targets both Raf and VEGF and PDGF receptor tyrosine kinase signaling. Mol Cancer Ther 2008;7:3129-40.

67. Gadaleta-caldarola G, Infusino S, Divella R, et al. Sorafenib: 10 years after the first pivotal trial. Fut Oncol 2015;11:1863-80.

68. Raskopf E, Dzienisowicz C, Hilbert T, et al. Effective angiostatic treatment in a murine metastatic and orthotopic hepatoma model. Hepatology 2005;41:1233-40.

69. Abou-Alfa GK, Schwartz L, Ricci S, et al. Phase II study of sorafenib in patients with advanced hepatocellular carcinoma. J Clin Oncol 2006;24:4293-300.

70. Ge S, Huang D. Systemic therapies for hepatocellular carcinoma. Drug Discov Ther 2015;9:352-362.

71. Deng G, Zeng S, Shen H. Chemotherapy and target therapy for hepatocellular carcinoma : New advances and challenges. World J Hepatol 2015;7:787-98. 